\title{
The Preparation of a Highly Stretchable Cellulose Nanowhisker Nanocomposite Hydrogel
}

\author{
Jiufang Duan, ${ }^{1}$ Jianxin Jiang, ${ }^{1}$ Jianzhang Li, ${ }^{1}$ Liujun Liu, ${ }^{1}$ Yiqiang Li, ${ }^{2}$ and Chao Guan ${ }^{2}$ \\ ${ }^{1}$ MOE Engineering Research Center of Forestry Biomass Materials and Bioenergy, Beijing Forestry University, Beijing 100083, China \\ ${ }^{2}$ Beijing Shoufa Tianren Ecological Landscape Co., Ltd., Beijing 102600, China \\ Correspondence should be addressed to Jiufang Duan; duanjiu99@163.com
}

Received 30 October 2014; Revised 12 January 2015; Accepted 21 January 2015

Academic Editor: Ning Lin

Copyright (c) 2015 Jiufang Duan et al. This is an open access article distributed under the Creative Commons Attribution License, which permits unrestricted use, distribution, and reproduction in any medium, provided the original work is properly cited.

\begin{abstract}
Molecules that associate to form cross-links by hydrophobic association are designed and synthesised. Hydrogels, based on cellulose nanowhiskers (CNWs), acrylamide (AM), and stearyl methacrylate (C18), were synthesised by micellar copolymerisation, using ammonium peroxydisulfate as an initiator. CNWs composite hydrogels were characterised by Fourier transform infrared spectroscopy (FTIR) and their morphologies were investigated by scanning electron microscope (SEM). The system shows the original extensibility up to about $2500 \%$ : the tensile strength and compressive strength have maximum values of $1.338 \mathrm{MPa}$ and 2.835 MPa, respectively. Besides excellent mechanical properties, CNWs composite hydrogels also have the ability to self-heal and remould: this is mainly attributed to the dissociation and reassociation of the associated micelles. In contrast to conventional cellulose hydrogels, these systems, when broken or cut, can be simply repaired by bringing together fractured surfaces to self-heal at room temperature.
\end{abstract}

\section{Introduction}

The preparation and application of hydrogels based on cellulose have been reviewed by some groups, because of their applications across several technologies, such as hygienic products, horticulture, gel-actuators, drug delivery systems, water blocking tapes, and coal dewatering [1-5]. Cellulose, as the most abundant renewable source on earth, offers an answer to maintaining hydrogel materials' sustainable development as an economically and ecologically attractive technology. Although a wide range of applications have been proposed for such hydrogels due to their unique properties, most hydrogels suffer from a lack of strength and self-healing ability [1].

An attractive method of designing multifunctional hydrogels is to use the concept of supramolecular polymers. Nanocomposite gels are a useful strategy when seeking to improve hydrogel mechanical strength. CNWs have attracted much attention, not only because of their unsurpassed quintessential physicochemical properties, but also because of their inherent renewability and sustainability in addition to their abundance. They have been the subject of a wide array of research projects as reinforcing agents in nanocomposites due to their low cost, nanoscale dimension, renewability, availability, and unique morphology [6]. Various nanocomposite gels with high mechanical strength have been widely investigated [7-9]. Considering the excellent properties of CNWs, the fabrication, moulding, and application of hydrogels containing CNWs have many advantages compared with other solid nanocomposite systems $[10,11]$. The application of such gels is typically limited by their poor mechanical properties: CNWs used for the reinforcement of polyacrylamide matrices have attracted much attention [11,12]; however, multifunctional hydrogels reinforced with CNWs are less well-studied $[13,14]$. Cellulose hydrophobically associating hydrogel with high mechanical strength and self-healing ability has seldom been reported.

Here, we report a new type of CNWs composite hydrogel that can overcome the aforementioned shortcomings. The reported CNWs composite hydrogel is a hydrophobic association hydrogel, which was prepared by micellar copolymerisation. AM and CNWs acted as the main monomer to 
form a hydrophilic backbone. C18, dodecyl 2-methylacrylate (C12), and tridecyl methacrylate (C13), respectively, acted as hydrophobic monomers to form associated micelles that are the physical cross-linking points in a network of CNWs composite hydrogels: because of the unique network structure, CNWs composite hydrogels exhibit excellent strength and rubber-like properties, the most remarkable properties being that CNWs composite hydrogels are self-healing.

\section{Experimental Work}

2.1. Materials and Methods. Acrylamide, sodium dodecylsulfate (SDS), stearyl methacrylate, ammonium persulfate (APS), $\mathrm{N}, \mathrm{N}, \mathrm{N}, \mathrm{N}^{\prime}$-tetramethylethylenediamine (TEMED), tridecyl methacrylate, dodecyl 2-methylacrylate, and $\mathrm{NaCl}$ were commercially available and used as received.

IR spectra were recorded by FTIR (Nicolet iN10 Thermo Fisher Scientific China) over the region from 4000 to $400 \mathrm{~cm}^{-1}$. The CNW structure was analysed by atomic force microscopy (Shimadzu, SPM-9600).

For morphological characterisation, the hydrogels were analysed by scanning electron microscope (SEM) (S-3400N, Hitachi, Japan) with an acceleration voltage of $40 \mathrm{kV}$. A thin layer of the sample was cast on a silica wafer and freezedried, overnight, in a lyophiliser. A layer of gold was sputtercoated over the sample by vacuum spray to form a conductive surface.

Images of CNWs were obtained using atomic force microscopy in intermittent contact mode. Samples for AFM were prepared by placing a drop of dilute CNW suspension on freshly cleaved mica, followed by rinsing in deionised water and drying under a gentle flow of $\mathrm{N}_{2}$.

Tensile stress-strain measurements were performed by using an Instron 3365 Universal Testing Machine (Norwood, MA, USA) with the following parameters: sampling rate, $10.000 \mathrm{pts} / \mathrm{sec}$; beam speed, $100 \mathrm{~mm} / \mathrm{min}$; full scale load range, $0.1000 \mathrm{kN}$; humidity, $25 \%$; and temperature, $24^{\circ} \mathrm{C}$. The strip-shaped gel samples measured $100 \mathrm{~mm} \times 10 \mathrm{~mm} \times$ $3 \mathrm{~mm}$ and the original length between top and foot clamps was $25 \mathrm{~mm}$. Each data point was measured on six samples, and the average value of five measurements was taken. Statistical analysis of data was performed by one-way analysis of variance, assuming a confidence level of $95 \%(P<0.05)$ for statistical significance.

The gravimetric method was used to measure the swelling ratios of the gels. After immersion in distilled water for approximately $48 \mathrm{hr}$ at $25^{\circ} \mathrm{C}$ to reach swelling equilibrium, the gel samples were weighed. The average value of three measurements was taken. The equilibrium swelling ratio (SR) was calculated as $\mathrm{SR}=W_{s} / W_{d}$, where $W_{s}$ is the weight of the swollen gel and $W_{d}$ is the weight of the gel in its dry state.

\subsection{Preparation of CNW Nanocomposite Hydrogels}

2.2.1. Preparation of CNWs. The cotton fibres were firstly soaked in dimethyl sulphoxide $(60 \mathrm{~mL})$ for $6 \mathrm{~h}$ at room temperature. After this, the fibres were washed in deionised water. The CNWs were obtained through acidic hydrolysis of the cotton fibres using 45 wt. $\% \mathrm{H}_{2} \mathrm{SO}_{4}$ (a cellulose $/ \mathrm{H}_{2} \mathrm{SO}_{4}$ ratio of $1 / 20 \mathrm{~g} / \mathrm{mL}$ ) at $75^{\circ} \mathrm{C}$ for $10 \mathrm{~h}$ under vigorous magnetic stirring. After this process, the resulting solution was centrifuged at 10,000 rpm for $5 \mathrm{~min}$ and washed thoroughly with deionised water until a $\mathrm{pH}$ of 7 was reached. The resultant material was lyophilised at $57^{\circ} \mathrm{C}$ for $48 \mathrm{~h}$.

2.2.2. Synthesis of CNW Nanocomposite Hydrogels. The gels were synthesised by micellar copolymerisation. The reaction system generally consisted of CNWs, water-soluble monomers (AM), hydrophobic monomers (C12, C13, or C18), surfactants (SDS), and water: SDS $(0.7 \mathrm{~g})$ and $\mathrm{NaCl}(0.30 \mathrm{~g})$ were dissolved in $9.9 \mathrm{~mL}$ of dispersion of CNWs at $35^{\circ} \mathrm{C}$ to obtain a transparent solution. Then, hydrophobic monomer C18 (0.09 g, 4.3 wt.\% relative to the amount of solid content) was dissolved in this solution under stirring for $2 \mathrm{~h}$ at a temperature of $35^{\circ} \mathrm{C}$. After adding and dissolving AM ( $0.90 \mathrm{~g}$, $45.9 \mathrm{wt} . \%$ relative to the amount of solid content) for $30 \mathrm{~min}$, TEMED $(25 \mu \mathrm{L})$ was added to the solution. Finally, $0.08 \mathrm{~g}$ of APS was added to initiate the reaction. The copolymerisation reactions were carried out at $25^{\circ} \mathrm{C}$ for $24 \mathrm{~h}$.

\section{Results and Discussion}

3.1. Synthesis of CNWs Composite Hydrogels. CNWs can be isolated from various renewable sources (wood, cotton, wheat/rice straw, etc.). Figure 1 shows AFM images of cottonderived CNWs. The cotton-derived CNWs have a rod-like morphology (diameter: 10 to $67 \mathrm{~nm}$, length: 100 to $250 \mathrm{~nm}$ ). The main process for the isolation of CNWs from cellulose fibres is based on acid hydrolysis. The cotton cellulose chains have a more ordered structure, with a crystallinity of about $70 \%$ [5]. The amorphous regions appear as imperfections in the cellulose microfibrils [5]. The crystalline regions of cellulose having a higher resistance to acid attack remain intact: disordered regions in the cellulose are preferentially hydrolysed. Followed by an acid treatment that hydrolyses the cellulose, colloidal particle CNWs were obtained [5].

We used CNWs, AM, and metacrylic acid ester (C12, $\mathrm{C13}$, or $\mathrm{C} 18$ ) as reactor monomers and added SDS to form solubilised micelles or comicelles with the hydrophobic monomers in an aqueous solution. Copolymerisation was initiated by ammonium persulfate herein, and the hydrogels thus obtained were transparent and resilient (Figure 1).

In this reaction system, CNWs and AM acted as the main monomer to form a hydrophilic backbone. C12, C13, and C18 acted as hydrophobic monomers to form associated micelles (Figure 1) that form the physical cross-linking points in a network of cellulose gels. The salt $(\mathrm{NaCl})$ led to micellar growth and solubilisation of large hydrophobes within the asgrown worm-like SDS micelles [15]. However, the micelles were stable enough to avoid destruction under the applied conditions.

3.2. Characterisation of the CNWs Composite Hydrogels. The structure of hydrogels of acrylamide with different hydrophobic monomers (stearyl methacrylate (a), tridecyl methacrylate (b), or dodecyl 2-methylacrylate (c)) and CNWs were 


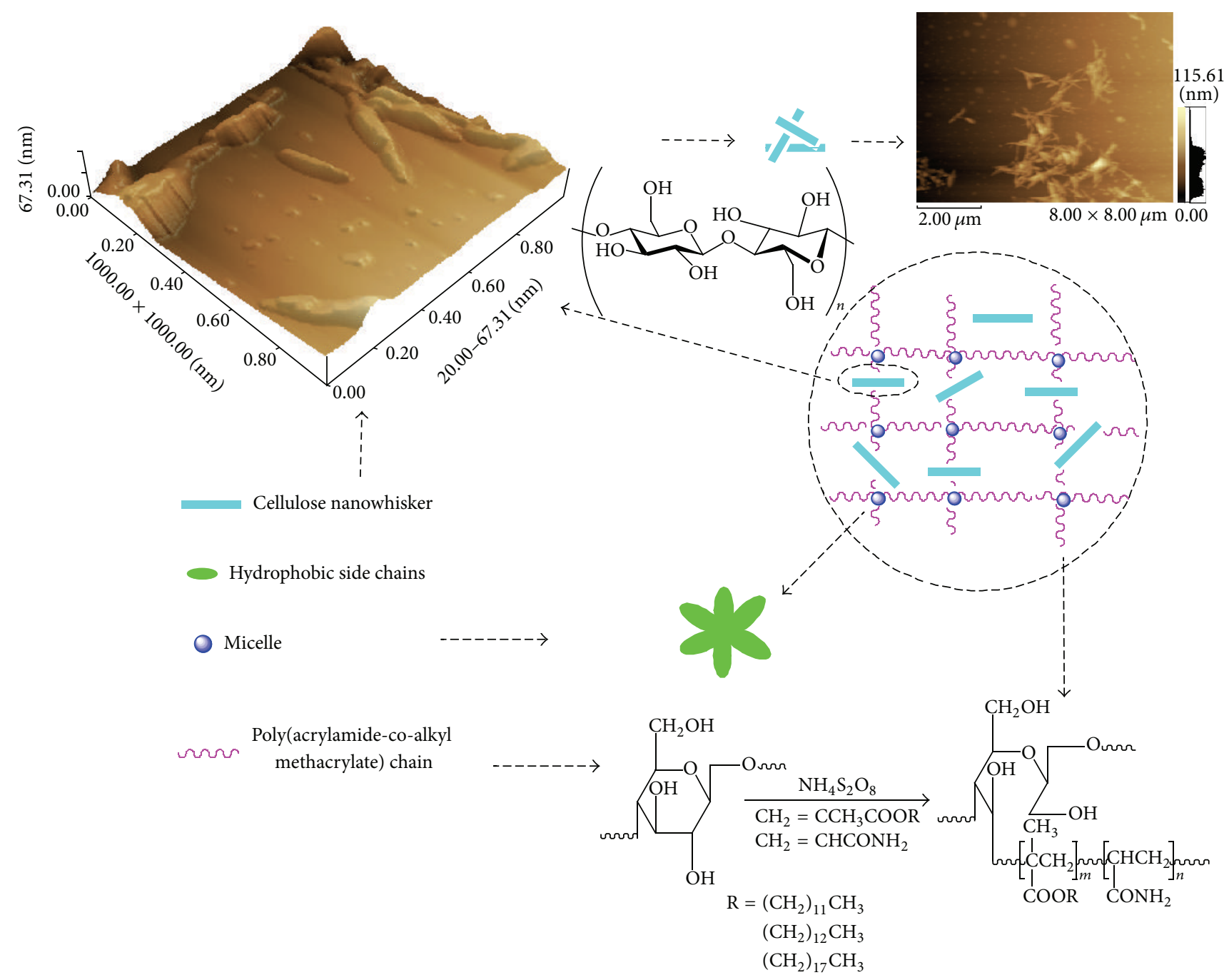

FIGURE 1: Scheme of the CNWs composite hydrogel matrix.

characterised by FTIR (Figure 2). It was observed that the hydrogen bond stretched at $3345 \mathrm{~cm}^{-1}$. The $\mathrm{C}-\mathrm{O}-\mathrm{C}, \mathrm{C}-\mathrm{C}-$ $\mathrm{O}$, and $\mathrm{C}-\mathrm{C}-\mathrm{H}$ deformation modes and stretching vibrations, in which the motions of the C-5 and C-6 atoms are at $898 \mathrm{~cm}^{-1}$, and the $\mathrm{C}-\mathrm{OH}$ out-of-plane bending mode around $670 \mathrm{~cm}^{-1}$ also occurred. According to Figure 2, absorption was observed at $1730 \mathrm{~cm}^{-1}$ (stretching vibration, $\mathrm{C}=\mathrm{O}$ ), $1060 \mathrm{~cm}^{-1}$ (asymmetrical stretching vibration of the ether bond), $2940 \mathrm{~cm}^{-1}$ and $2830 \mathrm{~cm}^{-1}$ (stretching vibration, C$\mathrm{H}$ bond in methylene), $1651 \mathrm{~cm}^{-1}$, and $792 \mathrm{~cm}^{-1}$ (bending vibration, $\mathrm{N}-\mathrm{H}$; stretching vibration, $\mathrm{C}-\mathrm{N})$. The intensity of the stretching vibration of methylene increased with the increase of the carbon chain length of the hydrophobic monomer.

\subsection{Mechanical Properties of the CNWs Composite Hydrogels.} The effect of the concentration of cellulose, acrylamide content, and content (and types) of hydrophobic monomers on the gel mechanical properties was analysed. First, we

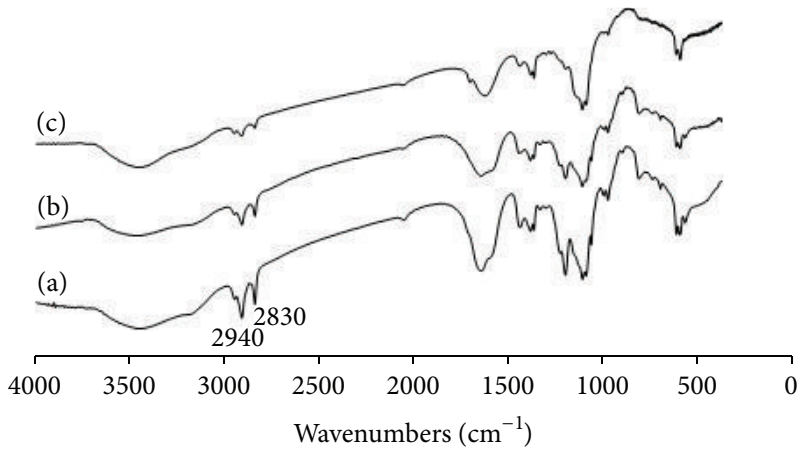

FIGURE 2: FTIR spectra of CNWs composite hydrogels (stearyl methacrylate (a), tridecyl methacrylate (b), or dodecyl 2-methylacrylate (c)).

synthesised the gel at the same concentration and experimental conditions with only $\mathrm{CNW}$ concentration changed 


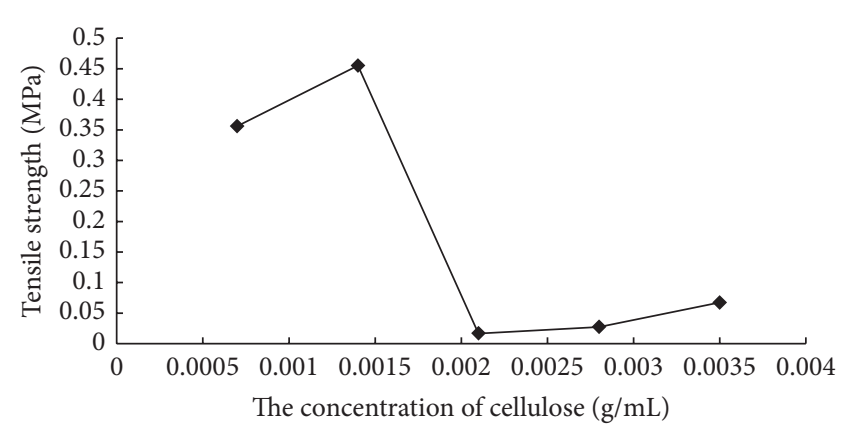

(a)

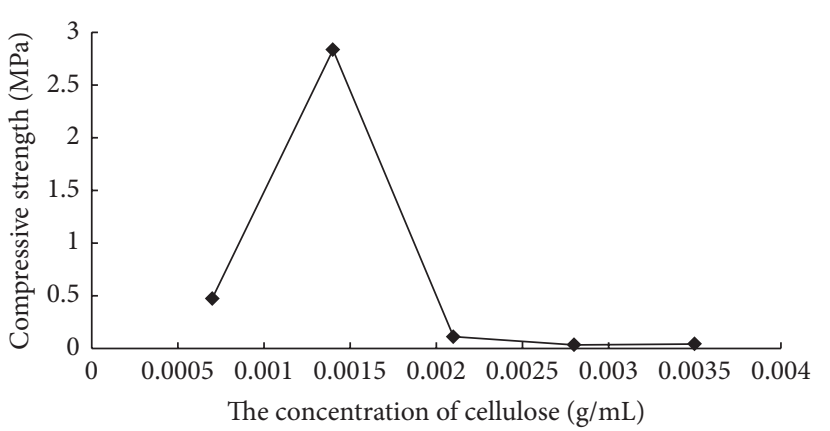

(b)

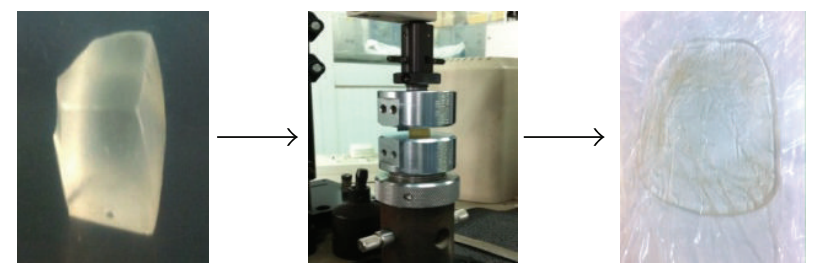

(c)

FIGURE 3: Effect of CNW concentration on CNWs composite hydrogel mechanical properties ((a) tensile strength; (b) compressive strength; (c) compression strength test images of hydrogel with a CNW concentration of $0.0014 \mathrm{~g} / \mathrm{mL}$ ).

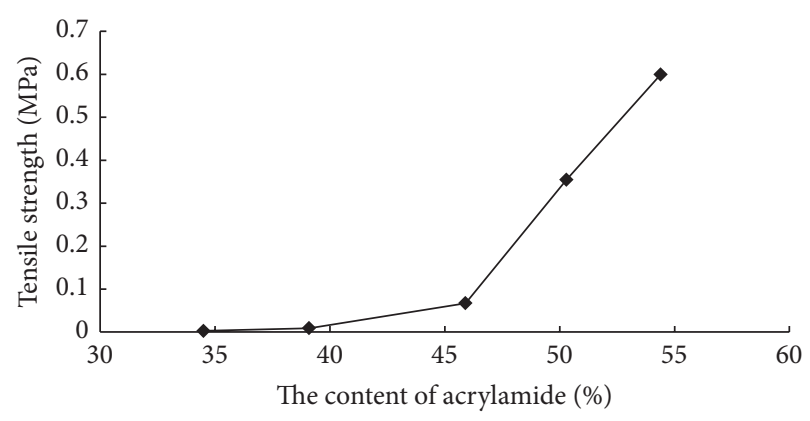

(a)

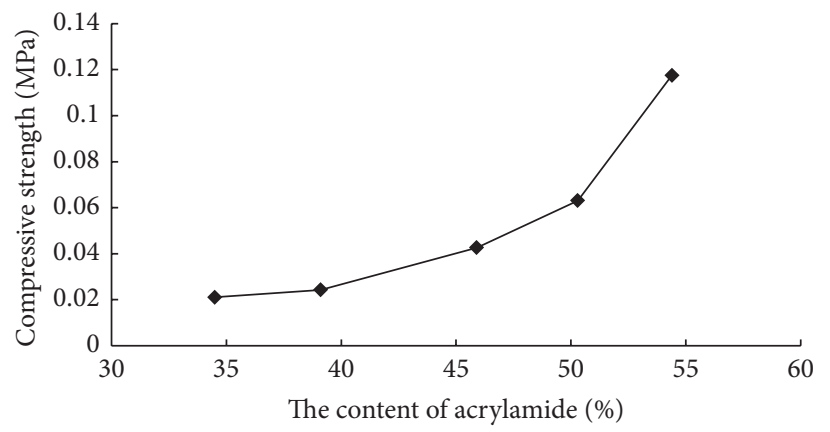

(b)

FIGURE 4: Effect of acrylamide content on CNWs composite hydrogel mechanical properties ((a) tensile strength and (b) compressive strength).

$(0.0007 \mathrm{~g} / \mathrm{mL}, 0.0014 \mathrm{~g} / \mathrm{mL}, 0.0021 \mathrm{~g} / \mathrm{mL}, 0.0028 \mathrm{~g} / \mathrm{mL}$, and $0.0035 \mathrm{~g} / \mathrm{mL})$.

Figure 3 shows the mechanical properties of the CNWs composite hydrogel specimens using $\mathrm{C} 18$ as hydrophobic monomer. When increasing the concentration of CNWs from $0.0007 \mathrm{~g} / \mathrm{mL}$ to $0.0035 \mathrm{~g} / \mathrm{mL}$, the tensile strength showed a maximum value of $0.4550 \mathrm{MPa}$ and a compressive strength of $2.8 \mathrm{MPa}$ at a cellulose concentration of $0.0014 \mathrm{~g} / \mathrm{mL}$. A possible reason is that high-aspect ratio fibres have the ability to sustain mechanical stress in a uniform manner. When the cellulose content used exceeds the critical concentration thereof, it will be difficult to avoid aggregation during their dispersion in the matrix because of their tendency to entangle. In principle, nonhomogeneous filler dispersion caused by entanglement usually results; this, in turn, leads to poor composite properties and an inefficient strengthening effect. The hydrogel cannot be crushed as shown in Figure 3(c) (a hydrogel with a CNW concentration of $0.0014 \mathrm{~g} / \mathrm{mL}$ ).

We synthesised gel of $\mathrm{C} 18$ under the same concentration and experimental conditions with only the dosage of AM changed from $34.5 \mathrm{wt} . \%$ to $54.4 \mathrm{wt}$.\%. Figure 4 shows the mechanical properties of those gel specimens using $\mathrm{C} 18$ as hydrophobic monomer. With increasing amounts of AM, the tensile strength and compressive strength increased from $0.0028 \mathrm{MPa}$ to $0.5998 \mathrm{MPa}$ and $0.0211 \mathrm{MPa}$ to $0.1175 \mathrm{MPa}$, respectively. The increased tensile and compressive strength were consistent with the expected increases in cross-linking densities in the three-dimensional network structure. As a result, the intertwining of polymeric chains was promoted and the hydrogen-bonding interaction between hydrophilic groups such as $-\mathrm{CONH}_{2}$ and $-\mathrm{OH}$, among others, was strengthened. Thus, the degree of cross-linking increased, a fact that was favourable to the improvement of mechanical 


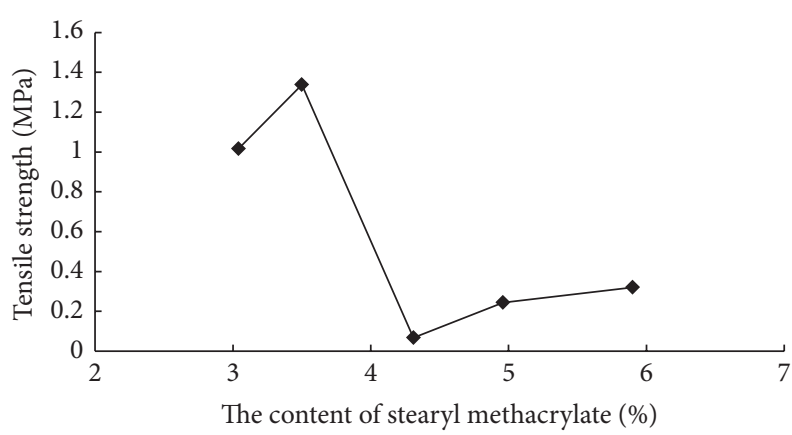

(a)

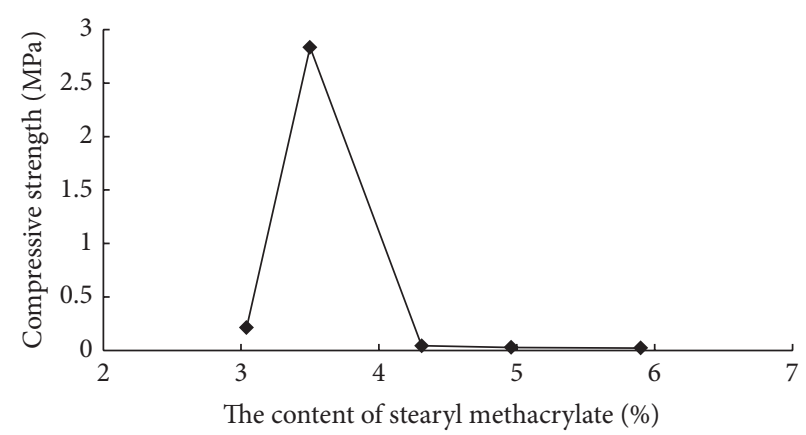

(b)

FIGURE 5: Effect of stearyl methacrylate content on CNWs composite hydrogel mechanical properties ((a) tensile strength and (b) compressive strength).

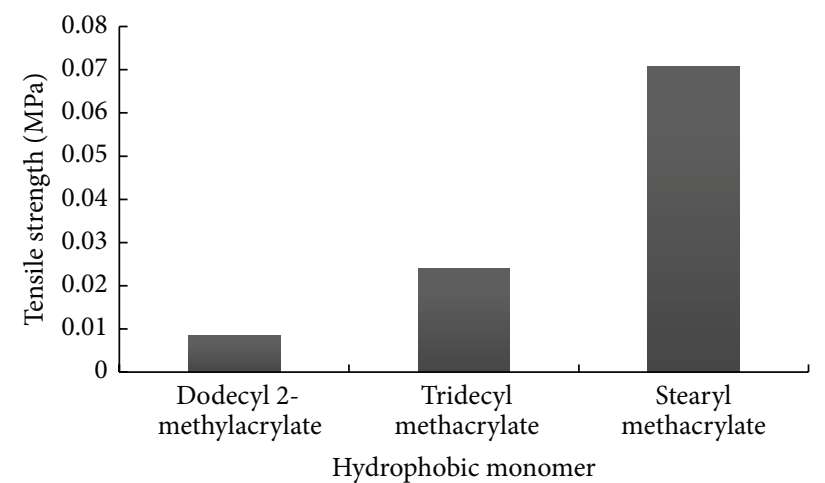

(a)

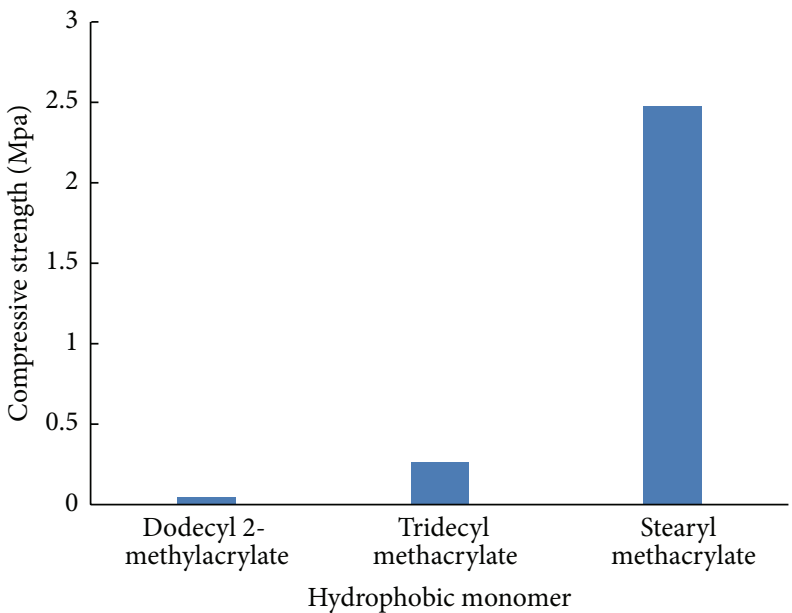

(b)

FIGURE 6: Effect of hydrophobic monomer on CNWs composite hydrogel mechanical properties ((a) tensile strength and (b) compressive strength).

properties. The same influences will also arise with increasing cross-link density in traditional polymers cross-linked by chemical bonds.

Figure 5 shows the mechanical properties of those gel specimens using $\mathrm{C} 18$ as hydrophobic monomer. The tensile and compressive strengths had maximum values of $1.338 \mathrm{MPa}$ and $2.835 \mathrm{MPa}$, respectively, when the C18 content was $3.50 \mathrm{wt} . \%$; then, with further increases in the amount of hydrophobic groups, the tensile and compressive strengths decreased. It seemed that the action of hydrophobic groups on polymer chains was similar to that on the chemical crosslinking points [16].

Figure 6 shows the mechanical properties of gel specimens using $\mathrm{C} 12, \mathrm{C} 13$, and $\mathrm{C} 18$ as hydrophobic monomer. We synthesised gels with hydrophobic monomer C12, C13, and C18 under identical experimental conditions. With increasing hydrophobic side chain length, the tensile, and compressive, strengths increased from $0.0084 \mathrm{MPa}$ to $0.0708 \mathrm{MPa}$ and from $0.0483 \mathrm{MPa}$ to $2.4800 \mathrm{MPa}$, respectively. The reason for this was that the hydrophobic side chain length became larger while the intermolecular hydrophobic interaction became stronger. In other words, increasing the length of hydrophobic side chain between backbones will induce an improvement in the mechanical properties of these gels.

3.4. Surface Morphology of CNWs Composite Hydrogels. Figure 7 shows SEM micrographs of freeze-dried nanocomposite hydrogel filled with cellulose nanowhiskers $(0.0007 \mathrm{~g} /$ $\mathrm{mL}, 0.0014 \mathrm{~g} / \mathrm{mL}, 0.0021 \mathrm{~g} / \mathrm{mL}, 0.0028 \mathrm{~g} / \mathrm{mL}$, and $0.0035 \mathrm{~g} /$ $\mathrm{mL}$ ), after being swollen to equilibrium. It was seen that the hydrogels had a heterogeneous pore distribution and it seemed that the addition of CNWs would change the morphology. CNWs composite hydrogel (Figure 7(a), $0.0007 \mathrm{~g} /$ $\mathrm{mL}$ ) had a relatively large-pored structure as well as the CNWs composite hydrogel (Figure 7(e), $0.0035 \mathrm{~g} / \mathrm{mL}$ ) which had a relatively dense surface structure. This may have been caused by the different intermolecular forces arising from the alteration of the cellulose content. This kind of surface allows a higher influx of water into the polymeric matrix, which would then benefit the water absorbency by such superabsorbent composites. 


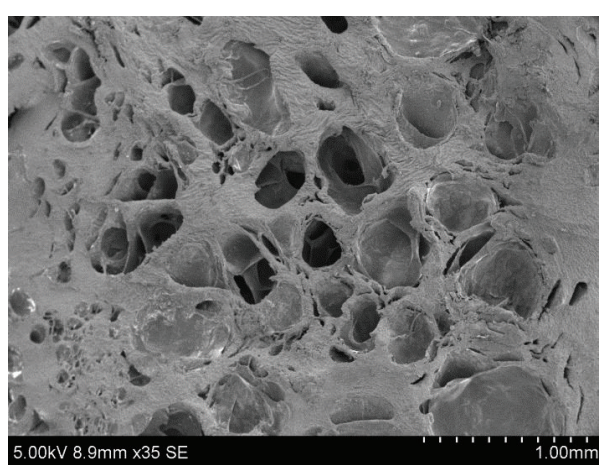

(a)

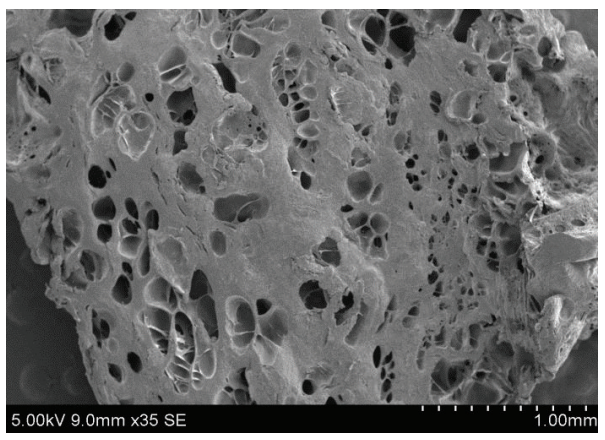

(c)

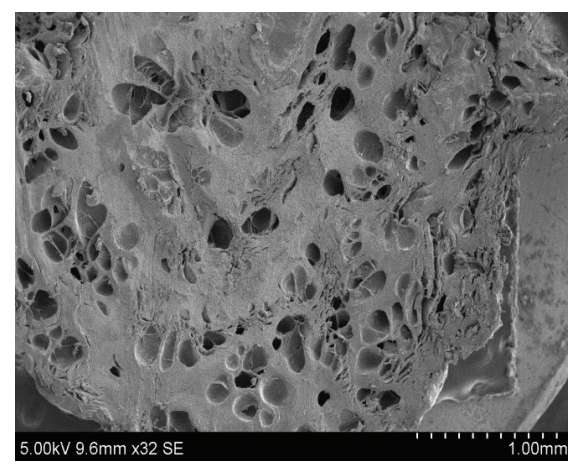

(b)

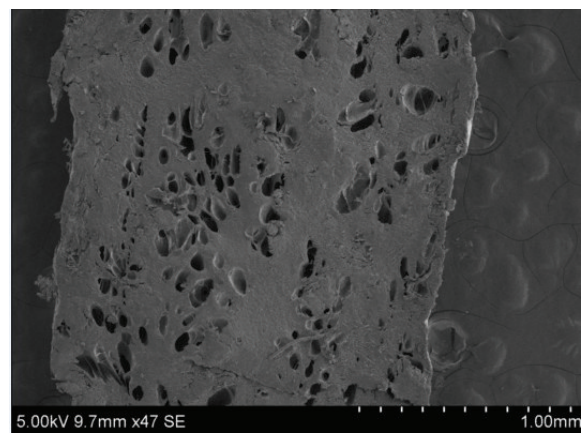

(d)

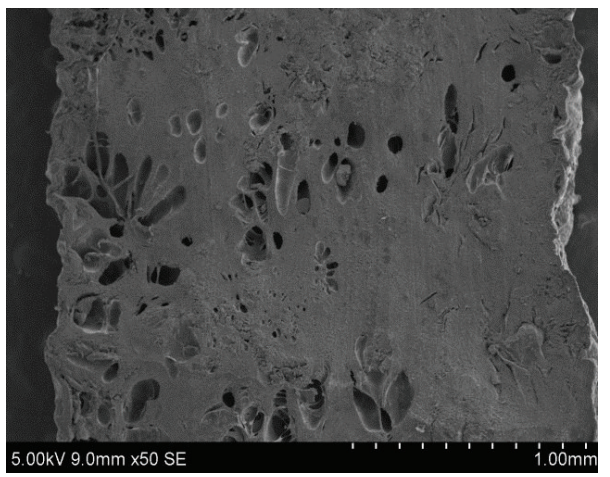

(e)

Figure 7: Scanning electron microscopy of CNWs composite hydrogels ((a) cellulose concentration of $0.0007 \mathrm{~g} / \mathrm{mL}$, (b) cellulose concentration of $0.0014 \mathrm{~g} / \mathrm{mL}$, (c) cellulose concentration of $0.0021 \mathrm{~g} / \mathrm{mL}$, (d) cellulose concentration of $0.0028 \mathrm{~g} / \mathrm{mL}$, and (e) cellulose concentration of $0.0035 \mathrm{~g} / \mathrm{mL}$ ).

3.5. The SR of the CNWs Composite Hydrogels. Hydrogels can absorb large amounts of water and release the absorbed water in dry conditions. A decrease in the water uptake capacity of these CNWs composite hydrogels was observed (Figure 8). It could be seen that the incorporation of $0.0007 \mathrm{~g} / \mathrm{mL}$ of CNWs produced the maximum water uptake in CNWs composite hydrogels. However, greater amounts of CNWs decreased the availability of functional groups from the matrix that were able to interact with the water; correspondingly the interaction between hydrophilic groups from the filler and the hydrogel network caused a decline in osmotic swelling pressure; finally the influx of water into the hydrogel matrix decreased [11]. Furthermore, the excessive amount of CNWs particles may physically stack in the network voids, decreasing the water-holding capability: consequently, the hydrogels can absorb less water as the CNW content increased [11].

3.6. The Self-Healing Behaviour of CNWs Composite Hydrogels. CNWs composite hydrogels exhibit excellent strength and rubber-like properties in a unique network structure (Figure 1): the most remarkable properties are that cellulose gels have the ability to self-heal (Figure 9). After cutting a gel sample into two parts and then bringing them together for $60 \mathrm{~min}$ at room temperature, it was found that the cut had healed (Figure 9). Not only can it be shape-formed in a polymerisation system, but also it would be reformed by 


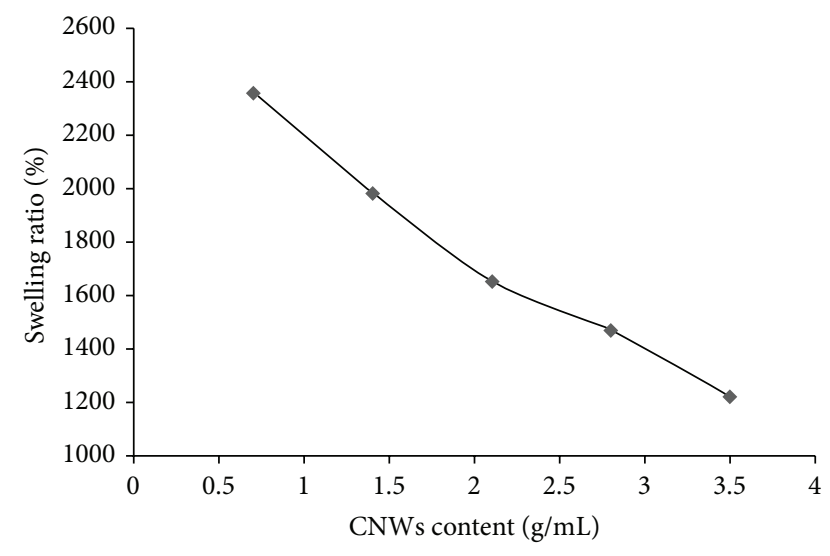

FIGURE 8: Swelling ratios of CNWs composite hydrogels.

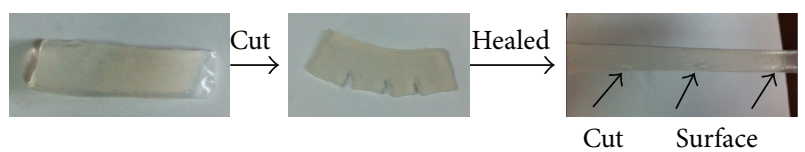

FIGURE 9: Self-healing behaviour of CNWs composite hydrogels.

a remoulding process as thermoplastic resins would if the process continued for sufficient time. This behaviour was ascribed to the rearrangement of cross-linking structures by dissociation and reassociation of hydrophobic groups. This endowed these gels with the ability to self-heal and be remoulded in a fashion similar to a thermoplastic resin.

Gels with SDS exhibited a self-healing efficiency of nearly $100 \%$ after a healing time of $60 \mathrm{~min}$. However, when swollen in water, no self-healing ability was observed. This was maybe because the gels swelled in water leading to the extraction of SDS micelles from the gel network: the surfactant SDS controlled the hydrophobic associations formed in the hydrogels. This suggested that the key factor leading to self-healing was the weakening of strong hydrophobic interactions due to the presence of surfactant molecules [17].

\section{Conclusions}

A simple method of making CNWs composite hydrogels that are transparent and soft materials containing a large amount of water has been proposed. In many applications, the use of hydrogels is often severely limited by their mechanical properties. Using CNWs to prepare "smart" hydrogels could not only improve their self-healing and reforming capacity but also improve their mechanical strength. CNWs composite hydrogels exhibited excellent mechanical properties; both the tensile strength and the compressive strength strongly depended on hydrophobic monomer content, hydrophobic side chain length, and AM and cellulose contents. C18 in the CNWs composite hydrogels played an important role in the domination of the physical cross-linking points formed by hydrophobic association, leading to self-healing and reforming capacity, whereas CNWs and AM mainly contributed to the increasing equilibrium between mechanical strength and swelling ratio thereof. Their unique self-repairing properties, the simplicity of their synthesis, their availability from renewable resources, and the low cost of their raw ingredients (cellulose) bode well for future applications.

\section{Conflict of Interests}

The authors declare that there is no conflict of interests regarding the publication of this paper.

\section{Acknowledgments}

This paper is supported by " 2015 Beijing Construction Project of Scientific Research and Cultivation of Graduate Student" and "Research Program of Circulation Utilization of Plant Waste 2014HXKFCLXY034.”

\section{References}

[1] J.-Y. Sun, X. Zhao, W. R. K. Illeperuma et al., "Highly stretchable and tough hydrogels," Nature, vol. 489, no. 7414, pp. 133-136, 2012.

[2] M. M. Abeer, M. C. I. M. Amin, A. M. Lazim, M. Pandey, and C. Martin, "Synthesis of a novel acrylated abietic acid-g-bacterial cellulose hydrogel by gamma irradiation," Carbohydrate Polymers, vol. 110, no. 1, pp. 505-512, 2014.

[3] X. Qiu and S. Hu, “'Smart' materials based on cellulose: a review of the preparations, properties, and applications," Materials, vol. 6, no. 3, pp. 738-781, 2013.

[4] R. Cha, Z. He, and Y. Ni, "Preparation and characterization of thermal/pH-sensitive hydrogel from carboxylated nanocrystalline cellulose," Carbohydrate Polymers, vol. 88, no. 2, pp. 713718, 2012.

[5] A. Sannino, C. Demitri, and M. Madaghiele, "Biodegradable cellulose-based hydrogels: design and applications," Materials, vol. 2, no. 2, pp. 353-373, 2009.

[6] Y. Habibi, L. A. Lucia, and O. J. Rojas, "Cellulose nanocrystals: chemistry, self-assembly, and applications," Chemical Reviews, vol. 110, no. 6, pp. 3479-3500, 2010.

[7] K. Haraguchi and T. Takehisa, "Nanocomposite hydrogels: a unique organic-inorganic network structure with extraordinary mechanical, optical, and swelling/de-swelling properties," Advanced Materials, vol. 14, no. 16, pp. 1120-1124, 2002.

[8] L. Xiong, X. Hu, X. Liu, and Z. Tong, "Network chain density and relaxation of in situ synthesized polyacrylamide/hectorite clay nanocomposite hydrogels with ultrahigh tensibility," Polymer, vol. 49, no. 23, pp. 5064-5071, 2008.

[9] J. Yang, C.-R. Han, J.-F. Duan et al., "Synthesis and characterization of mechanically flexible and tough cellulose nanocrystalspolyacrylamide nanocomposite hydrogels," Cellulose, vol. 20, no. 1, pp. 227-237, 2013.

[10] C. Zhou, Q. Wu, Y. Yue, and Q. Zhang, "Application of rodshaped cellulose nanocrystals in polyacrylamide hydrogels," Journal of Colloid and Interface Science, vol. 353, no. 1, pp. 116123, 2011.

[11] C. Spagnol, F. H. A. Rodrigues, A. G. V. C. Neto et al., "Nanocomposites based on poly(acrylamide-co-acrylate) and cellulose nanowhiskers," European Polymer Journal, vol. 48, no. 3, pp. 454-463, 2012. 
[12] F. H. A. Rodrigues, C. Spagnol, A. G. B. Pereira et al., "Superabsorbent hydrogel composites with a focus on hydrogels containing nanofibers or nanowhiskers of cellulose and chitin," Journal of Applied Polymer Science, vol. 131, no. 2, Article ID 39725, pp. 1-13, 2014.

[13] M. Larsson, Q. Zhou, and A. Larsson, "Different types of microfibrillated cellulose as filler materials in polysodium acrylate superabsorbents," Chinese Journal of Polymer Science, vol. 29, no. 4, pp. 407-413, 2011.

[14] M. Harini and A. P. Deshpande, "Rheology of poly(sodium acrylate) hydrogels during cross-linking with and without cellulose microfibrils," Journal of Rheology, vol. 53, no. 1, pp. 3147, 2009.

[15] D. C. Tuncaboylu, M. Sari, W. Oppermann, and O. Okay, "Tough and self-healing hydrogels formed via hydrophobic interactions," Macromolecules, vol. 44, no. 12, pp. 4997-5005, 2011.

[16] G. Jiang, C. Liu, X. Liu, G. Zhang, M. Yang, and F. Liu, "Construction and properties of hydrophobic association hydrogels with high mechanical strength and reforming capabilitya," Macromolecular Materials and Engineering, vol. 294, no. 12, pp. 815-820, 2009.

[17] D. C. Tuncaboylu, M. Sahin, A. Argun, W. Oppermann, and O. Okay, "Dynamics and large strain behavior of self-healing hydrogels with and without surfactants," Macromolecules, vol. 45, no. 4, pp. 1991-2000, 2012. 

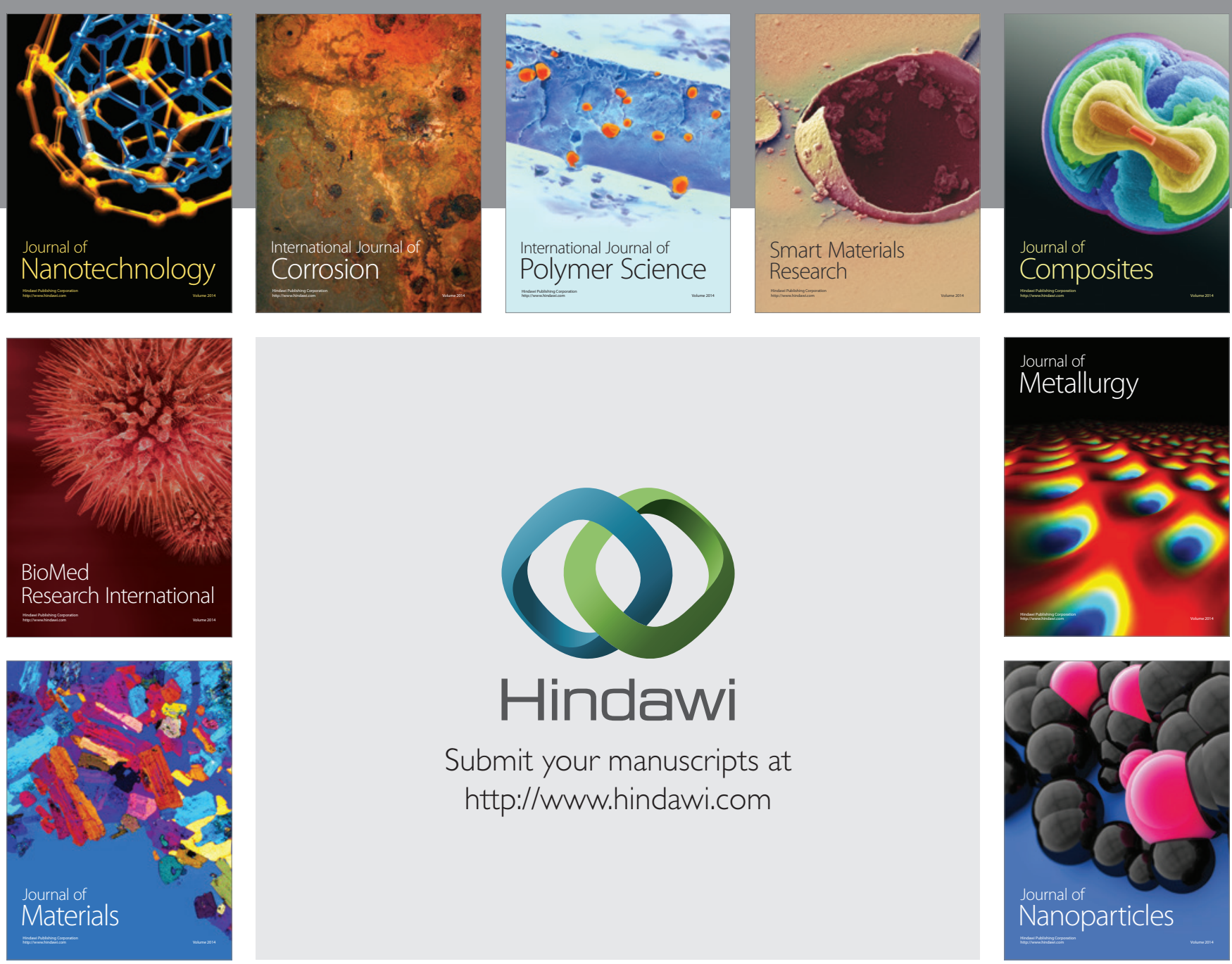

Submit your manuscripts at http://www.hindawi.com
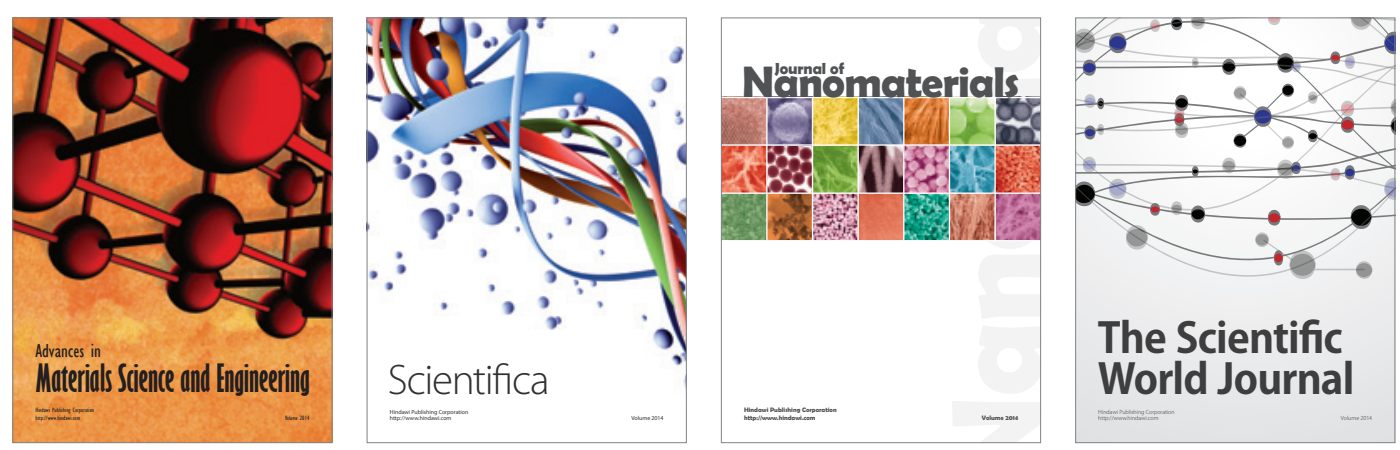

\section{The Scientific World Journal}
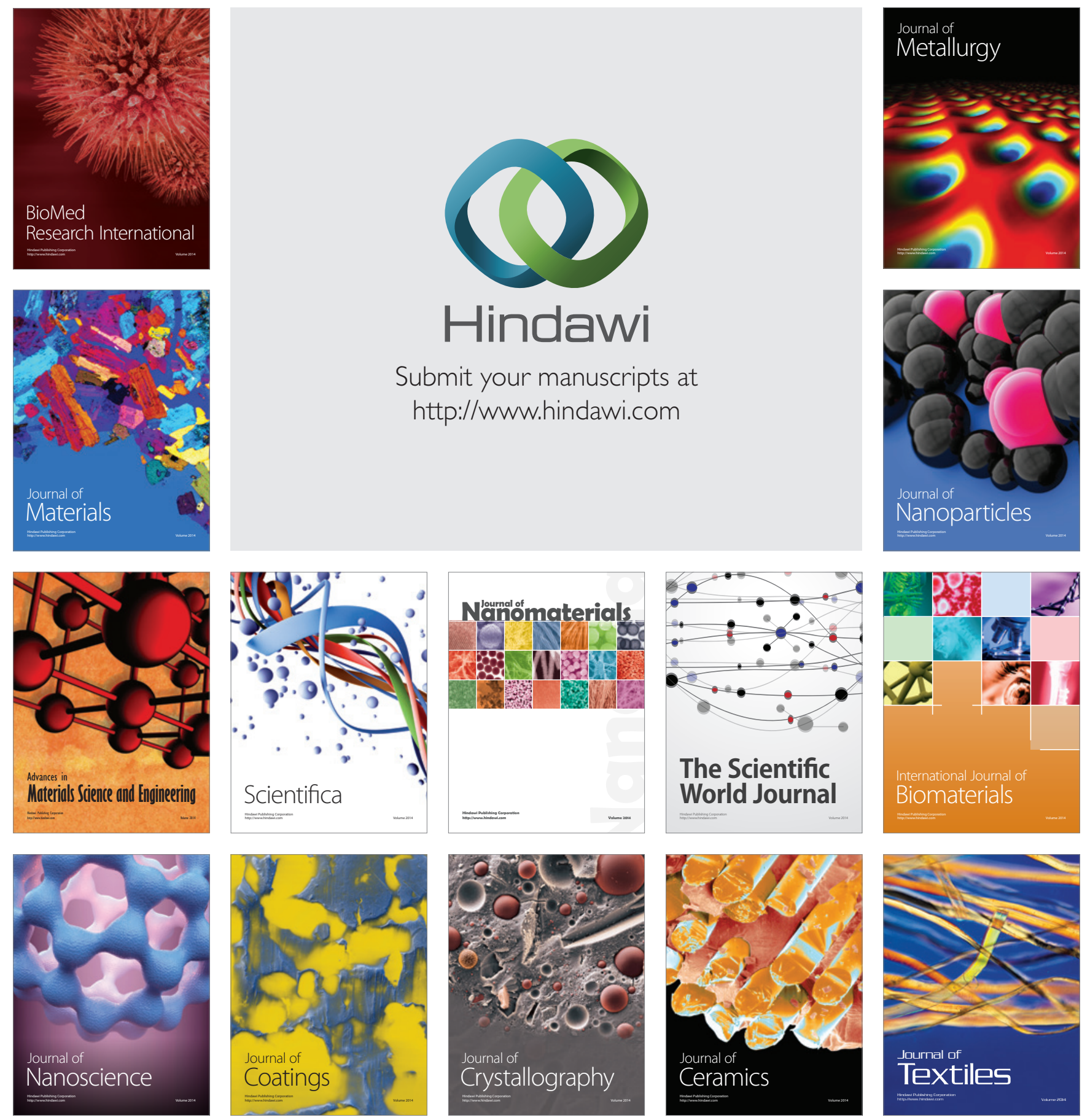